$16^{\text {th }}$ International Congress of Metrology, 05006 (2013)

DOI: $10.1051 /$ metrology/201305006

(C) Owned by the authors, published by EDP Sciences, 2013

\title{
Construction of a large graphite calorimeter for measurements in small fields used in radiotherapy
}

\author{
Stéphane Dufreneix ${ }^{1, a}$, Jean-Marc Bordy ${ }^{1}$, Josiane Daures ${ }^{1}$, Frank Delaunay ${ }^{1}$ and Aimé Ostrowsky ${ }^{1}$ \\ ${ }^{1}$ CEA, LIST, Laboratoire National Henri Becquerel (LNE-LNHB), 91191 Gif-sur-Yvette CEDEX, France
}

\begin{abstract}
As the national metrology institute for ionization radiation the LNE-LNHB develops, maintains and transfers dosimetric references for high energy x-ray radiotherapy beams. Through the European project MetrexRT, a new approach has recently been developed to extend the dosimetric references to small fields (less than $2 \mathrm{~cm}$ wide). This method is based on the measurement of an absorbed dose on a surface larger than the beam. A new primary dosimeter (a graphite calorimeter) with a large surface has been built and characterized as well as a large surface ionization chamber. Both dosimeters can now be considered operational for measurements in small beams.
\end{abstract}

\section{Introduction}

High energy x-ray beams (4-21 MeV) are commonly used in radiotherapy to treat cancer. The aim of this technique is to focus $\mathrm{x}$-rays on the tumor in order to kill its cells by an energy deposition. The quantity of interest is the absorbed dose to water defined as the mean energy deposited by $\mathrm{x}$-rays in a volume of known mass (expressed in Gray ie $\mathrm{J}_{\mathrm{kg}}{ }^{-1}$ ). Both spatial precision and dose determination are of great importance for the success of a radiotherapy treatment.

Thanks to technological advances, field sizes used in radiotherapy have been decreasing down to a few millimeters which enables a more precise delivery of the dose. Nevertheless this gain in spatial resolution is linked to an increase in the uncertainty on the delivered dose, due to specific phenomena occurring when field size gets smaller than $2 \mathrm{~cm} \mathrm{[1-3].}$

The reference conditions for the determination of the absorbed dose in radiotherapy are defined in international protocols: measurements are conducted in a $10 \mathrm{~cm} \mathrm{x} 10$ $\mathrm{cm}$ field at $10 \mathrm{~cm}$ depth in a water tank phantom [4-5]. Recently, the LNE-LNHB also established dosimetric references in $4 \mathrm{~cm} \mathrm{x} 4 \mathrm{~cm}$ and $2 \mathrm{~cm} \times 2 \mathrm{~cm}$ beams [6] by diminishing the size of the dosimeters [7] in order to keep them fully exposed. For smaller field sizes, a new approach has been suggested based on the use of large dosimeters to measure the absorbed dose deposited by the irradiation beam on its surface [8-9] (Figure 1).

As part of the European project in charge of the development of this method, the LNE-LNHB constructed a new graphite calorimeter (named GR11) to establish primary dose references in small fields. Its originality lies in its dimensions: the core has a diameter of $30 \mathrm{~mm}$, nearly twice the size of the former GR09 used in the 10 $\mathrm{cm} \times 10 \mathrm{~cm}$ reference field (16 $\mathrm{mm}$ diameter). A plateparallel ionisation chamber with a collecting electrode of $30 \mathrm{~mm}$ has also been built to become the reference chamber used for transfers to users.

\section{Construction of the GR11}

\subsection{Generalities about graphite calorimetry}

Calorimetry is the most fundamental method to establish absorbed dose references in high energy x-ray beams [10]. Because the sensitive volume has to be large and precisely defined, only graphite calorimetry can be used in the approach considered here. It has a very good sensitivity thanks to its low specific heat capacity and its atomic number is close to the one of water. Nevertheless it needs some procedures to get to the absorbed dose to water.

All graphite calorimeters at LNE-LNHB have the same structure described in $[7,11]$. They are made of 3 bodies: a core, a jacket and a shield. The absorbed dose in the core can be derived from the heat elevation which occurs under irradiation. However, this heat elevation is quite small: 1 Gy deposited leads to roughly $1 \mathrm{mK}$ temperature elevation. That's why the jacket and the shield which surround the core need to be regulated in temperature. The shield is set at a temperature slightly higher than the room's temperature to be independent of its fluctuations. The jacket acts like a buffer between the shield and the core. To minimize convection transfer, all bodies are separated by a vacuum interstice $\left(10^{-3} \mathrm{~Pa}\right.$ at the entry of the pump).

\footnotetext{
${ }^{\mathrm{a}}$ Corresponding author: stephane.dufreneix@cea.fr
} 
Figure 1. Establishment of dosimetric references in broad and small fields (sensitive volumes are hatched).

$10 \mathrm{~cm} \times 10 \mathrm{~cm}$

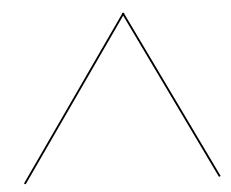

$\mathbb{Z Z Z}$
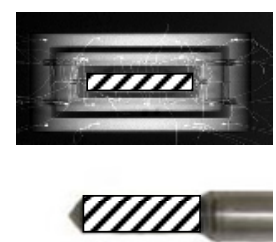

$4 \mathrm{~cm} \times 4 \mathrm{~cm}$

$2 \mathrm{~cm} \times 2 \mathrm{~cm}$
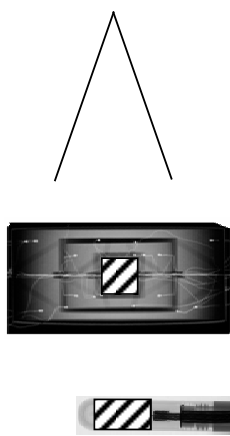

\begin{tabular}{|c|c|}
\hline Calorimeter GR10 & $\emptyset: 6 \mathrm{~mm}$ \\
\hline $\begin{array}{c}\text { Ionisation Chamber: } \\
\text { Exradin A1SL }\end{array}$ & $\begin{array}{c}\mathrm{V}: 0.057 \\
\mathrm{~cm}^{3}\end{array}$ \\
\hline
\end{tabular}

$<2 \mathrm{~cm}$

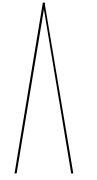

$\mathbb{Z W W W Z}$
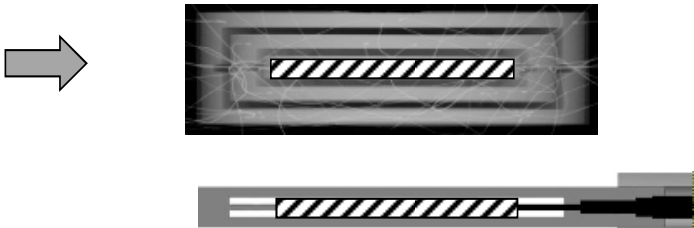

\begin{tabular}{|c|c|}
\hline Calorimeter GR11 & Ø: $30 \mathrm{~mm}$ \\
\hline Ionisation Chamber: & Ø: $30 \mathrm{~mm}$ \\
\hline
\end{tabular}

\subsection{Thermistors}

Thermistors are electronic components which resistance is directly linked to the temperature using equation (1)

$$
\mathrm{R}_{\mathrm{th}}(\mathrm{T})=\mathrm{R}_{0} * \exp \left[-\beta\left(1 / \mathrm{T}-1 / \mathrm{T}_{0}\right)\right]
$$

where $\mathrm{R}_{0}$ is the resistance of the thermistor at $\mathrm{T}_{0}=298.15$ $\mathrm{K}$ and $\beta$ is the sensitivity index.

Thermistors are used in a graphite calorimeter either to measure the temperature of a body or to heat it. For GR11, 26 thermistors were used: 6 in the core, 10 in the jacket and 10 in the shield. For each body, 2 thermistors are used for temperature measurements, the others for heating. BR14 thermistors from General Electric Sensing (Figure 2) were chosen for their small dimension (glass bulb of $0.41 \mathrm{~mm}$ diameter) and the small diameter of their wire $(0.28 \mathrm{~mm})$ which limits conduction between the bodies.

Figure 2. Photograph of a thermistor BR14.

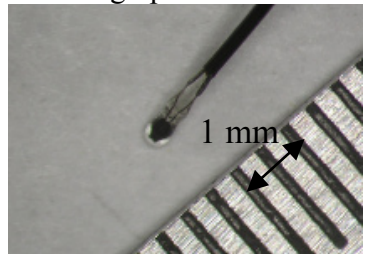

The characteristics of 250 thermistors were measured in order to choose those with similar $R_{0}$ and $\beta$. This selection is necessary to later consider that heat is uniformly distributed in any of the 3 bodies during heating.

$\mathrm{R}_{0}$ and $\beta$ were determined at LNE-LNHB by measuring the resistance $R_{\text {th }}$ of each thermistor placed in water at 3 different temperatures. A calibrated thermistor $\left(\mathrm{R}_{0}=8486 \pm 5 \Omega\right.$ and $\left.\beta=3356 \pm 3 \mathrm{~K}\right)$ was used to determine T. A linear fit between $\ln \left(\mathrm{R}_{\mathrm{th}}\right)$ and $1 / \mathrm{T}$ gave $\beta$ and $\ln \left(\mathrm{R}_{0}\right)$. The dispersion on $\mathrm{R}_{0}$ and $\beta$ could thus be decreased from $15 \%$ given by the manufacturer to less than $0.5 \%$ for each group of thermistors.

\subsection{Assembly}

In order to minimize radiation transfers between the bodies, the faces of the jacket and the shield were covered with a $7 \mu \mathrm{m}$ aluminised mylar which has a high reflection coefficient. No mylar was glued on the core's surface in order to avoid the introduction of materials different from graphite. Those are indeed considered as impurities and have to be taken into account later by a correction factor.

Thermistors were glued into the bodies. The core was suspended and centred inside the jacket with 3 kapton tubes. Thermistors wires were then bent and positioned in the groves of the jacket. The same steps were followed for the assembly of the jacket in the shield. A radiography was realised to check the quality of the final assembly (Figure 3).

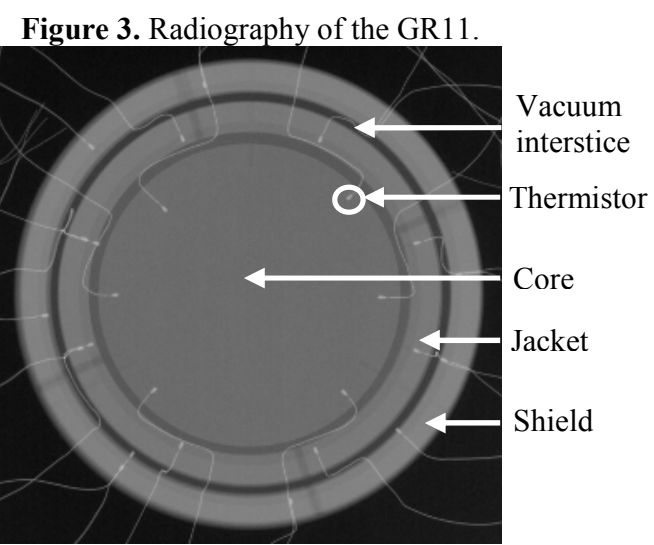

\subsection{Characteristics}

\subsubsection{Mass of the core}

The determination of the mass of the core is essential to deduce the absorbed dose from the heat elevation that occurs under irradiation in the core. 
The core is thermally composed of the graphite slab, the 6 thermistors, the glue used during the assembly, the supporting kapton tubes, the thermistors' wires inside the graphite slab and half of the kapton tubes and thermistors' wires between the core and the jacket. It is assumed that there is a perfect thermal contact between the tubes/wires and the core on one side and the jacket on the other side. The mass of this body has been determined by adding the separated mass of these different components (Table 1). All weightings were compared to a calibrated mass and corrected for aerostatic pressure.

Table 1. Mass and composition of the core.

\begin{tabular}{|l|ccc|}
\cline { 2 - 4 } \multicolumn{1}{c|}{} & Mass $(\mathrm{mg})$ & Uncertainty $(\mathrm{mg})$ & $\%$ \\
\hline Graphite & 3773.968 & \pm 0.05 & $99.65 \%$ \\
6 thermistors & 6.648 & \pm 0.30 & $0.18 \%$ \\
3 kapton tubes & 0.595 & \pm 0.05 & $0.01 \%$ \\
Glue & 6.050 & \pm 0.09 & $0.16 \%$ \\
\hline Core & 3787.26 & \pm 0.32 & $100.00 \%$ \\
\hline
\end{tabular}

\subsubsection{Thickness}

The calorimeter is placed inside of a vacuum case composed of a PMMA ring and 2 graphite blocks sealed with a $0.1 \mathrm{~mm}$ thick mylar sheet. The linear thickness of the entire system is $16.0 \pm 0.2 \mathrm{~mm}$ for each side and the corresponding mass thicknesses are $2.34 \pm 0.01{\mathrm{~g} . \mathrm{cm}^{-2}}^{-2}$ for the front face and $2.33 \pm 0.01 \mathrm{~g} . \mathrm{cm}^{-2}$ for the back face. Dosimetric measurements can be conducted at different depths with several graphite slices of various thicknesses.

\subsubsection{Resistance of the wires $R_{w}$}

Due to Joule effect, some heat power is lost in the thermistors' wires even if this loss is not linked to a temperature variation of the core. This can later lead to a systematic error in measurements.

As $\mathrm{R}_{\text {th }}$ (resistance of the thermistor) depends on the temperature but not $\mathrm{R}_{\mathrm{w}}$, the difference in dose measured at different temperatures can be attributed to $R_{w}$ and a correction can be applied (Figure 4).

Figure 4. Dose rates measured with and without correction of the resistance of the wires $R_{w}$.

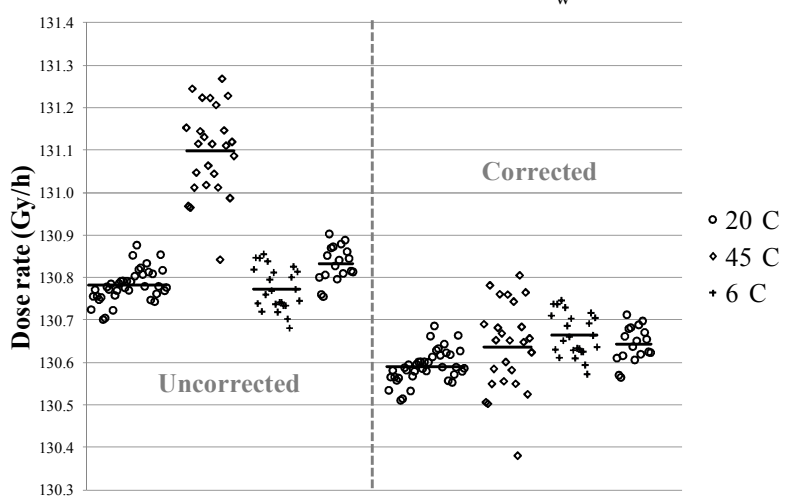

Measurements conducted with the GR11 stabilized at 3 different temperatures $\left(6,20\right.$ and $\left.45^{\circ} \mathrm{C}\right)$ gave $\mathrm{R}_{\mathrm{w}}=$ $13.3 \pm 0.7 \Omega$ and the correction applied ranges from 0.08 $\%$ to $0.36 \%$ depending on the temperature.

\subsubsection{Heat leakages between the bodies}

Heat leakages between the core and the jacket and between the jacket and the shield can be calculated theoretically with the equations of convection, conduction and radiation transfers. They can also be evaluated when each body of the calorimeter is regulated at a given temperature by changing the thermal regulation point of the jacket. This modifies the temperature difference between the bodies and thus heat leakage between the core and the jacket can be deduced from the power variation in the core whereas heat leakage between the jacket and the shield can be deduced from the sum of the power variations in the core and in the jacket (Figure 5).

Figure 5. Power variation in the bodies of the calorimeter when the temperature of the jacket is modified and the temperature of the core and shield are kept constants.

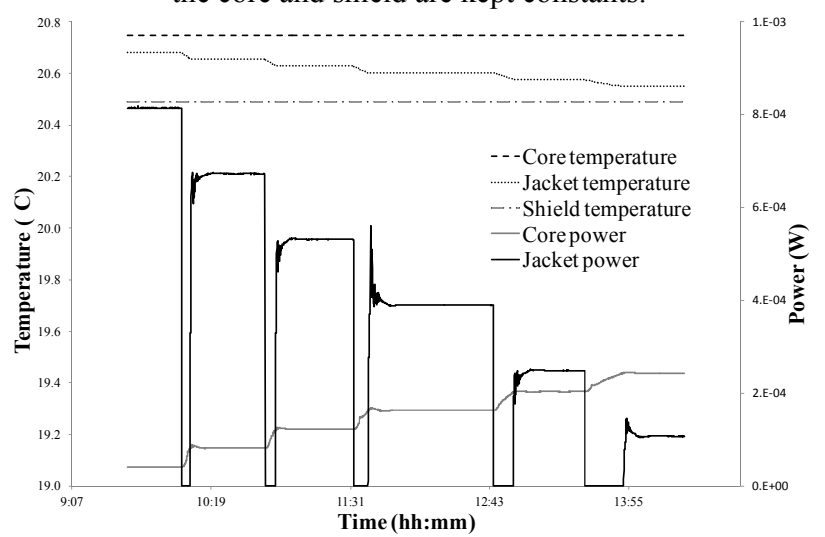

Results for GR11 are given in Table 2 and are compared with those of GR09 which has much smaller dimensions (diameter of the core: $16 \mathrm{~mm}$ ) and smaller thermistors' wires (diameter: $0.025 \mathrm{~mm}$ ), limiting heat leakages. Differences between theory and experiments are due to approximations on the geometry of the bodies and on the emissivity and thermal conductivity values taken.

Table 2. Heat leakages between the different bodies (in W.K ${ }^{-1}$ ).

\begin{tabular}{|l|l|c|c|}
\cline { 3 - 3 } \multicolumn{2}{l|}{} & Core-Jacket & Jacket-Shield \\
\hline \multirow{2}{*}{ GR11 } & Experiment & $1.5310^{-3}$ & $3.8410^{-3}$ \\
& Theory & $1.5110^{-3}$ & $3.1710^{-3}$ \\
\hline \multirow{2}{*}{ Experiment } & $4.9010^{-4}$ & $6.4010^{-4}$ \\
& Theory & $4.8010^{-4}$ & $5.9010^{-4}$ \\
\hline
\end{tabular}

\section{Construction of a reference ionization chamber}

\subsection{Generalities}

Because absolute dose measurements with a calorimeter are delicate and time-consuming, an ionisation chamber is used to transfer the dosimetric reference established to users. This type of dosimeter measures the current induced by ionisations in an air volume which occur under irradiation. 
To measure a dose deposited on a surface and especially on a disc, a plate parallel ionization chamber is convenient. A $2 \mathrm{~mm}$ thick single air cavity ionization chamber was designed at LNE-LNHB with a collecting electrode of $30 \mathrm{~mm}$ diameter (Figure 6). This allows the measurement of the dose deposited on the same surface as the one of GR11's core.

Figure 6. Drawing of the single air volume ionization chamber (sensitive volume is hatched).

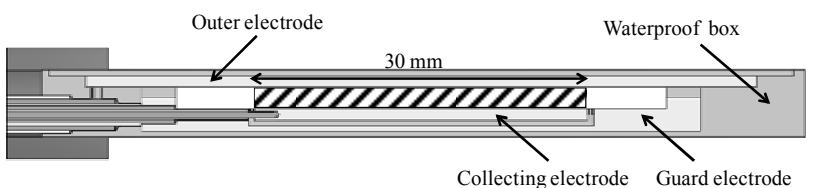

A double air cavity ionization chamber $(2 \times 1 \mathrm{~mm})$ with a collecting electrode of the same diameter in the middle was also considered but issues during the final step of the assembly made it unusable.

\subsection{Assembly}

The chamber was assembled after all components were polished and then washed with alcohol. A tri-axial cable was used: its core was glued inside the collecting electrode, the first shield was connected to the guard electrode and the second shield to the outer electrode. A PMMA waterproof box allows for measurements in water which is the reference medium in radiotherapy. The sensitive volume is connected to the ambient air through the support rod.

The background measured was less than $5.10^{-15} \mathrm{~A}$.

\subsection{Stability in water}

A reference ionization chamber has to be very stable in air as well as in water. At LNE-LNHB, a chamber is considered stable if deviation is less than $\pm 1 \%$ on $12 \mathrm{~h}$. This criterion was met in air but the first set of measurements in water with the single cavity chamber showed a strong deviation (Figure 7). After investigation, it appeared that micro water leaks were present where the sealing box had been glued. Once plugged, stability was much better. The residual deviation can be attributed to a variation of the air volume thickness due to deformation of the PMMA sealing box when put in water which can bring the electrodes closer $(5 \%$ deviation $=10 \mu \mathrm{m}$ thickness variation).

Figure 7. Deviation of the reference chamber in water.

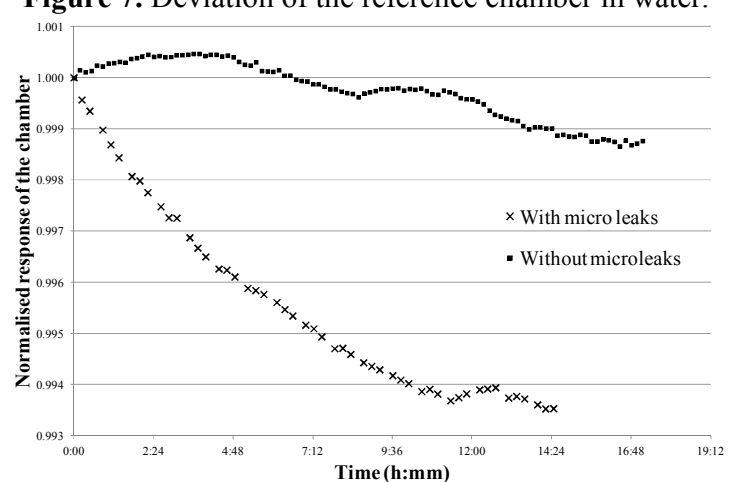

\section{Conclusions}

GR11 has been constructed and fully characterized. It will now be compared to other graphite calorimeters built at LNE-LNHB in the reference $10 \mathrm{~cm} \mathrm{x} 10 \mathrm{~cm}$ field which involves the determination of impurity correction factors.

The ionisation chamber can be used as a reference but to minimize the deviation in water, another chamber will be assembled with a sealing box made out of cross linked polystyrene which has a water absorption 10 times smaller than PMMA. Its response when applied high voltage varies will have to be studied in order to determine the polarity and the saturation correction factors.

The first step of the European project MetrexRT on metrology for radiotherapy using complex radiation fields is now nearly finished and measurements for establishing dosimetric references in small fields will begin in the following months.

\section{References}

1. Das, I.J. Ding, G.X. Ahnesjo, A. Med. Phys. 35 (1), 206-215 (2008)

2. Report Number 103, Small field MV photon dosimetry (Institute of Physics and Engineering in Medicine, York, 2010)

3. Rapport DPRH, Mesure de la dose absorbée dans les faisceaux de photons de très petite dimensions utilisés en radiothérapie stéréotaxique (Institut de Radioprotection et Sûreté Nucléaire / Société Française de Physique Médicale / Société Française de Radiothérapie Oncologique, 2008)

4. TRS 398, Absorbed dose determination in external beam radiotherapy: an international code of practice for dosimetry based on standards of absorbed dose to water (International Atomic Energy Agency, 2004)

5. Almond, P.R. Biggs, P.J. Coursey, B.M. Hanson, W.F. Huq, M.S. Nath, R. Rogers, D.W.O. Med. Phys. 26 (9), 1847-1870 (1999)

6. M. Le Roy, Etude des références dosimétriques nationales en radiothérapie externe - application aux irradiations conformationnelles (Thèse, Université de Nice - Sofia Antipolis, 2011)

7. Daures, J. Ostrowsky, A. Rapp, B. Metrologia 49 (5), S174-S178 (2012)

8. Djouguela, A. Harder, D. Kollhoff, R. Ruhmann, A. Willborn, K. C. Poppe, B. Zeitschrift Fur Medizinische Physik 16 (3), 217-227 (2006)

9. Ostrowsky, A. Bordy, J.M. Daures, J. De Carlan, L. Delaunay, F. Dosimetry for small size beams such as IMRT and stereotactic radiotherapy. Is the concept of the dose at a point still relevant? Proposal for a new methodology (Commissairiat à l'Energie Atomique Rapport R-6243, 2010)

10. Pruitt, J.S., S.R. Domen, and R. Loevinger, Journal of Research of the National Bureau of Standards 86 (5) 495-502 (1981)

11. Daures, J. Ostrowsky, A. Phys. in Med. and Biol. 50 (17), 4035-4052 (2005) 